\title{
Mast cell-related disorders presenting with Kounis syndrome
}

D. González-de-Olano; A. Matito; P. Sánchez-López; L. Sánchez-Muñoz; J.M. Morgado; C. Teodósio; M. Jara-Acevedo; A. García-Montero; A. Orfao; L. Escribano; N.G. Kounis; I. Álvarez-Twose

Systemic mastocytosis (SM) is a clinically heterogeneous group of disorders characterized by the accumulation of clonal $\mathrm{MC}$ in bone marrow (BM), and frequently also in other organs including the skin and/or the gastrointestinal tract. Currently, the clonal nature of the disease can be clearly established in most SM patients through the demonstration of somatic activating mutations involving the tyrosin-kinase regulatory domain of the Kit receptor. In most cases, such KIT mutations lead to continuously ligand-independent autophosphorylation of the receptor, and initiation of downstream signalling events resulting in the release of MC-mediators. In recent years, detailed clinical, biological and molecular characterization of MC activation syndromes (MCAS) not fulfilling enough criteria for SM has been made. Based on the presence or absence of KIT mutations, MCAS are currently classified into clonal MCAS (c-MCAS) and non-clonal MCAS (nc-MCAS). Unlike BM MC from SM patients, in which aberrant $C D 25$ expression and cytomorphological abnormalities, together with KIT mutations, are usually detected, BM MC from both c-MCAS and nc-MCAS cases frequently show a normal morphological appearance and/or immunophenotype. Patients with SM and MCAS suffer from systemic, usually recurrent symptoms, which are attributable to the release of MC mediators; Noteworthy, vasoconstriction-related features, such as increase of blood pressure, can be occasionally observed in a few patients during acute $M C$ activation episodes. Moreover, neurological ischemic symptoms suggesting a transient cerebral vasospasm have been recently reported in two patients diagnosed with indolent SM (ISM) and nc-MCAS, respectively. Altogether, these observations provide strong evidence that Kounis syndrome (KS) which denotes acute coronary events as a result mast cell mediator action might also occur in patients with MC-related disorders. Herein, we report a series of 10 patients with different MC-related disorders, who presented with acute coronary syndromes as an unusual manifestation of their MC-mediator symptomatology profile. To the best of our knowledge, this is the first series of KS presenting in patients with SM and MCAS reported so far. A total of 10 patients (six males and four females) with a median age of 57 years (range: 36 to 74 years) with a history of oppressive chest pain suggesting an ischemic heart event, who were referred to the Instituto de Estudios de Mastocitosis de Castilla La Mancha (CLMast) between July 2007 and March 2012 because of a suspected underlying MC disorder (e.g. SM or MCAS) based on clinical symptoms and/or serum tryptase levels, were included in the study.

At referral to the CLMast, patients were carefully explored for the presence of skin lesions suggestive of mastocytosis, and MC-mediator release symptoms were recorded. In addition, baseline serum tryptase (Phadia Diagnostics, Uppsala, Sweden) and total serum IgE levels were measured in all cases, while specific IgE (immunoCAP, Phadia Diagnostics) was investigated, at the time it was commercially available, in those cases in which triggers potentially causing an IgE-mediated reaction (e.g. food, insect stings or drugs), were identified. Specific serum IgE values $>0.35 \mathrm{KU} / \mathrm{L}$ were considered as positive. In order to properly classify the underlying MC disorder, a complete BM study was performed in 9/10 patients, following recently proposed criteria, which included assessment of BM MC morphology in toluidine blue and MayGrünwald-Giemsa stained smears, evaluation of the presence of focal dense BM MC aggregates (>15 MC per cluster) in BM sections stained for c-kit and tryptase, immunophenotypical analysis of CD25 and CD2 expression on BM MC by flow cytometry according to consensus criteria previously defined by the Spanish Network on Mastocytosis (REMA) and the investigation of somatic activating codon Asp816 $\rightarrow$ Val KIT mutation in genomic DNA from fluorescence-activated cell sorting-purified BM MC, performed as describe elsewhere. 
Diagnosis of SM was made strictly following the current World Health Organization (WHO) accepted criteria; by contrast, MCAS was diagnosed in cases not fulfilling enough criteria for SM, and MCAS patients were further subclassified as c-MCAS or nc-MCAS based on the presence VS. absence of mutated BM MCs, according to recent recommendations.

The clinical and laboratory findings of the 10 patients presenting with mast cell-related disorders and Kounis syndrome are shown in table 1.

In our series, ACS triggered by drugs, hymenoptera venom and foods was documented in most cases, which is in agreement with the most frequently observed causes of KS, reported in the literature. According to coronary arteriography findings, 3 of such patients were classified as type I variant $\mathrm{KS}$, one as type II variant and 4 cases remain unclassifiable because coronariography was not performed. In turn, the remaining two patients suffered from effortinduced ACS with no additional identifiable allergenic triggers in the setting of both an underlying atheromatous disease and a clonal MC disorder (ISM and c-MCAS, respectively). Although these two cases mimicked typical stable angina, a potential role of MC-mediator release in the genesis of ACS could not be ruled out, since exercise is an infrequent but wellrecognized trigger of $\mathrm{MC}$-mediator release in patients with anaphylaxis.

Increased tryptase levels (>11.4 $\mu \mathrm{g} / \mathrm{L}$ ) were detected in most cases included in our series. Unlike ISM cases, in which elevated baseline tryptase levels could be explained by chronic release by an increased number of MC, patients with MCAS usually have a low or even very low, MC burden. In this regard, it has been suggested that tryptase released by MC might contribute to control and prevent exaggerated allergic reactions through cleavage of IgE; whether this mechanism could also occur in patients with MCAS in between acute MCmediator release episodes, remains unknown.

Kounis syndrome is increasingly reported in clinical practice, but little is known about its association with $\mathrm{MC}$ disorders. Routine determination of serum tryptase levels during acute coronary symptoms might provide evidence for a potential role of $M C$ activation in the pathogenesis of ACS in a subset of patients. Further investigations are warranted in order to assess the utility of antimediator drugs in the management of these subjects. 